\title{
KAUFMANN, Thomas, Das Ende der Reformation. Magdeburgs « Herrgotts Kanzlei » (1548-1551/2)
}

\section{Philippe Büttgen}

\section{OpenEdition}

\section{Journals}

Édition électronique

URL : http://journals.openedition.org/ifha/872

DOI : $10.4000 /$ ifha. 872

ISSN : 2198-8943

Éditeur

IFRA - Institut franco-allemand (sciences historiques et sociales)

Référence électronique

Philippe Büttgen, «KAUFMANN, Thomas, Das Ende der Reformation. Magdeburgs « Herrgotts Kanzlei » (1548-1551/2) », Revue de l'IFHA [En ligne], Date de recension, mis en ligne le 01 janvier 2005, consulté le 22 septembre 2020. URL : http://journals.openedition.org/ifha/872 ; DOI : https://doi.org/10.4000/ ifha. 872

Ce document a été généré automatiquement le 22 septembre 2020.

(C)IFHA 


\title{
KAUFMANN, Thomas, Das Ende der Reformation. Magdeburgs « Herrgotts Kanzlei » (1548-1551/2)
}

\author{
Philippe Büttgen
}

1 Un moment, on pensa que c'en était fini de la Réforme, fini du monde aussi. À Mühlberg (24 avril 1547), l'Empereur avait défait les princes ligués à Schmalkalde ; à Augsbourg, l'année suivante, Charles Quint avait imposé à la Diète une Loi d'interim qui entendait faire taire les conflits religieux jusqu'à la fin du Concile en cours, mais ses dispositions, cultuelles surtout, eurent tôt fait de la transformer en loi d'exception contre les territoires protestants ; en Saxe, berceau luthérien, le duc Maurice, allié protestant de l'Empereur, avait ravi l'électorat à Jean-Frédéric, captif, et imposait depuis Leipzig son propre interim à sa population ; Wittenberg elle-même, son université et ses théologiens, s'étaient ralliés aux Albertins et travaillaient autour de Melanchthon à légitimer une politique d'attente et de médiation. Ceux qui refusèrent allèrent non loin, à Magdebourg : la ville, depuis 1524, avait embrassé la cause de la Réforme, et le conflit ancien entre le Conseil et l'Archevêché, dont Magdebourg relevait devant l'Empire, s'était spectaculairement accentué depuis qu'après Mühlberg la ville avait refusé de se soumettre au nouvel ordre impérial. Celui-ci triomphant à l'heure de la fin, il était tout juste encore temps de nommer les coupables et de confesser, une dernière fois, la saine doctrine. À Magdebourg, les réfugiés, assistés par les autorités locales, ouvrirent une " Chancellerie du Seigneur ", "Herrgotts Kanzlei », où, de pamphlet en feuille volante, de libelle en canard, on entendait assembler les actes de l'Antéchrist et les contre-preuves de la vraie foi.

2 L'objet du livre de Th.K. n'est pas l'Intérim, ni même Magdebourg, son siège par les troupes impériales et sa défaite finale en octobre 1551 : le lecteur devra reconstituer pour lui-même les repères chronologiques principaux, et gagnera à garder une histoire de la Saxe à portée de main. Pour peu qu'il consente à cet effort, Das Ende der Reformation lui fera tout voir autrement. Il s'agit ici d'une seule chose : la « Herrgotts Kanzlei » et ce qu'il en reste pour l'historien d'aujourd'hui, c'est-à-dire une masse de 
livres (de livres, à peine : de papier plutôt, mais de papier imprimé), d'où ressort un phénomène éditorial, politique, religieux, doctrinal, apocalyptique sans équivalent dans l'histoire moderne de l'Empire. Comment la résistance à l'Intérim transforma Magdebourg, l'espace de quatre années, en l'un des plus grands centres éditoriaux de l'Empire ; comment la coopération d'un groupe de théologiens fuyards, au premier rang desquels Matthias Flacius Illyricus, avec des magistrats épris de libertés municipales produisit l'une des plus formidables « mobilisations religieuses» (p. 91) de tout le XVIe s. ; comment l'attente eschatologique put s'installer au lieu géométrique d'une résistance théologico-politique et d'une « offensive de publication » (p. 57), tout cela est ici documenté comme jamais : la bibliographie de la production magdebourgeoise entre 1548 et 1552 est désormais complète et occupe 60 pages ; l'appareil graphique et statistique qui l'accompagne découragera l'habituelle critique "française » de la supposée abstraction " allemande »; et pour la première fois peut-être, l'eschatologie pré-moderne trouve le cadre de contextualisation qui permet de faire passer l'histoire avant la fascination - elle en avait, il est vrai, assez besoin.

On aurait donc envie de parler, à propos de la « Herrgotts Kanzlei », de « fait social total ", comme on le fait à chaque fois qu'un terrain d'enquête bien choisi permet d'associer plusieurs méthodes d'approche dont l'affinité était jusque là restée inaperçue. Il n'est pas sûr, toutefois, que l'intention de l'auteur soit à chercher de ce côté-là. Les premiers mots sont du reste tout aussi familiers à l'historien français : il s'agit d'enquêter sur Magdebourg comme « lieu de mémoire », locus memoriae creusé d'abord par un roman de Wilhelm Raabe, Unseres Herrgotts Kanzlei (1862), où le possessif vaut aussi pour l'appropriation, depuis lors, de Magdebourg par les consciences protestantes allemandes.

4 S'agissant de Magdebourg, la ville, le " lieu de mémoire " est à comprendre littéralement. Mais face à l'impressionnisme qui guette toute réminiscence, Th.K. propose encore une autre parade. Comme toute mémoire, celle de Magdebourg est partielle et abîmée ; comme mémoire d'une "Chancellerie du Seigneur ", elle se conserve éminemment dans ses produits : des livres et du papier proliférant. "Une ruine de mots » (Wortruine), donc, ce qui s'entend théologiquement, mais sans doute aussi militairement : pour les luthériens assiégés de Magdebourg, le « combat pour la Parole » (de Dieu) ne pouvait guère passer que par un « combat de paroles » (p. 40). Aujourd'hui, la ruine est faite des livres qui abritent ces paroles. La méthode de Th.K. s'impose de ne pas aller plus loin : chacune des « analyses de textes » (p.39) qui font le cœur de l'ouvrage est en fait l'analyse d'un livre ou d'un écrit entier (« integrale Sinneinheiten ", p. 209), qui s'attache aux contraintes du contexte et du genre de publication (dialogue, dispute académique, parodie, prêche, lettre ouverte, réimpression d'écrits adverses...). Par là sont évités les choix plus ou moins arbitraires de " thèmes » et de " motifs » dont se contente le plus souvent la reconstitution des mentalités « eschatologiques » de la première modernité. Il est vrai que le chapitre final sur les " contours du monde mental de la "Herrgotts Kanzlei" ", à propos de cette apocalyptique magdebourgeoise qui est à la fois déchiffrement de l'histoire et interprétation de soi, témoigne encore de la nécessité d'une "synthèse » (p. 431) qui sache, après la description, opérer la sélection. Restent l'élan d'un projet et la force d'une règle qui stipule de décoller le moins possible les mots du papier sur lequel ils ont d'abord été couchés : ailleurs qu'en Allemagne, on parlerait volontiers de " matérialité ", et l'on aurait tort de s'étonner de ce qu'en Allemagne même ce soit un historien de 
l'Église, que sa discipline a formé à l'archive, qui donne désormais la meilleure idée de ce que ce mot peut signifier.

5 Telle est en effet la nouveauté d'un livre qui par ailleurs aura autant de raisons d'étonner en France qu'en Allemagne : Th.K. pense, relate, explique Magdebourg au milieu des livres. Le linguistic turn n'a rien à voir ou presque avec cela, parce que les livres ici donnent moins accès à des mots qu'à des actions, celles que leurs auteurs ont souhaité accomplir en écrivant, en imprimant, en publiant. La chose est voulue par l'objet choisi, la " Herrgotts Kanzlei ", forme très remarquable d'intellectuel collectif dans laquelle l'imprimeur se tenait derrière l'auteur écrivant son libelle, et où l'auteur retrouvait l'imprimeur face aux presses, au moment de lui remettre le texte suivant. Il n'est pas demandé si cette forme s'est retrouvée ailleurs, si l'on peut en faire un type ou la rattacher à un modèle, celui par exemple du producteur de textes d'avant le tournant auctorial - question qui se pose par ailleurs d'une manière à la fois spécifique et pressante à propos du métier de théologien dans la première modernité. C'est bien plutôt la singularité de la " Herrgotts Kanzlei » qui intéresse Th.K., et ce que cette singularité permet d'ajouter aux débats en cours sur la périodisation de l'âge confessionnel (p. 13, sur la situation des Magdebourgeois "zwischen den Zeiten ", après la Réforme proprement dite, mais avant la confessionalisation), sur le républicanisme urbain dans l'Empire et les théories du droit de résistance (p. 180, en réponse aux recherches inspirées par Luise Schorn-Schütte, ou p. 193, sur le rôle historique toujours redébattu de la Confessio Magdeburgensis de 1550), sur la validité générale, enfin, du schéma de confessionnalisation (p. 101), la production de la " Herrgotts Kanzlei ", comme sa réception européenne (voir p. 103-118), servant de nouveau banc d'essai aux thèses de Th.K. sur la pluralité fragile du luthéranisme prémoderne et l'émergence simultanée d'une conscience " protestante » commune aux luthériens et aux réformés entre 1550 et 1650 (voir p. 486, ainsi que Dreißigjähriger Krieg und Westfälischer Friede. Kirchengeschichtliche Studien zur lutherischen Konfessionskultur, Tübingen : Mohr Siebeck, 1998).

6 Derrière le fait social total, derrière le lieu de mémoire, derrière les livres eux-mêmes, dans leur folie de défaite et d'attente, c'est peut-être alors un nouvel objet qui se profile. " Magdebourg », comme forteresse assiégée d'une doctrine qui se proclamait inflexiblement vraie, donne à réfléchir sur quelque chose que l'auteur nomme très tôt et dont il traite implicitement tout au long de l'enquête : un zèle ou un entêtement particuliers dans l'assertion doctrinale, une certaine disposition proclamatrice qui en langue originale se dit Beständigkeit (dès la p. 8), attachement inconditionnel à la " wahre Bekenntnis » (par ex. p. 170, 174), Beharrlichkeit (p. 437). Si la langue traite encore et toujours ces mots comme des valeurs - il en va ici de ce que " résister " pouvait vouloir dire pour des protestants du Saint-Empire -, il se pourrait en revanche que l'enquête entreprise sur la " Herrgotts Kanzlei ", en rencontrant à son tour dans son objet la " tendance 'pré-moderne' à la stabilisation des convictions acquises, au renforcement des identités, [...] à l'affirmation combative de soi »(p. 184-185), permette d'avancer vers une élucidation historique de ce qu'on pourra appeler, provisoirement, la modalité confessante du vrai dans la première modernité.

7 Un livre " 'allemand' ", alors, comme l'affirme l'auteur en prévenant d'entrée de son écrasante érudition (p. VIII) ? Outre les notes de bas de page, c'est tout l'appareil de démonstration qui porterait à acquiescer. Plus au fond, l'originalité provocante de l'objet, le choix non moins revendiqué de tirer de la matière tout ce qu'elle peut donner 
avant de commencer à théoriser, la radicalité, enfin, des résultats, inciteraient plutôt à abandonner les dénominations nationales et à parler simplement d'un grand livre d'histoire, aussi grand que singulier.

8 Philippe BÜTTGEN (Centre National de la Recherche Scientifique/ MHFA) 